\title{
Agentes hemostáticos tópicos em cirurgia: revisão e perspectivas.
}

\section{Topical hemostatic agents in surgery: review and prospects.}

\author{
Bruno Monteiro Pereira, ACBC-SP1; José Benedito Bortoto'; Gustavo Pereira Fraga, tCBC-SP1
}

R E S U M O

\begin{abstract}
A hemostasia tem papel crítico e importância fundamental em todos os procedimentos cirúrgicos. Seu manejo possui diversos pontos chaves, que se iniciam por boa técnica operatória e adequado suporte anestésico. Determinadas situações, como hemorragias graves resultantes de trauma penetrante, por exemplo, não dependem exclusivamente do controle da equipe cirúrgica e necessitam do apoio de novas soluções que diminuam ou controlem a hemorragia. Desde os tempos antigos, um marco da medicina é atuar no controle da hemorragia e, mais recentemente, na facilitação da hemostasia pela aplicação de agentes tópicos, seja por compressão manual ou agentes modernos. Na última década, o número de diferentes agentes hemostáticos tópicos cresceu drasticamente. Para que o cirurgião moderno escolha o agente correto no momento correto, é essencial que conheça o mecanismo de ação, entenda a eficácia e os possíveis efeitos adversos relacionados a cada agente. Assim, a grande variedade de hemostáticos tópicos, somada à ausência de um artigo de revisão na literatura nacional sobre este tópico, nos estimulou a elaborar este manuscrito. Aqui relatamos uma revisão detalhada sobre os agentes hemostáticos tópicos mais comumente utilizados nas especialidades cirúrgicas.
\end{abstract}

Descritores: Hemostáticos. Cirurgia Geral. Hemostasia Cirúrgica. Técnicas Hemostáticas. Procedimentos Cirúrgicos Operatórios.

\section{INTRODUÇÃO}

\begin{abstract}
hemostasia tem papel crítico e importância Afundamental em todos os procedimentos cirúrgicos. Seu manejo possui diversos pontos chaves, que se iniciam por boa técnica operatória e adequado suporte anestésico. Determinadas situações, como hemorragias graves resultantes de trauma penetrante, por exemplo, não dependem exclusivamente do controle da equipe cirúrgica e necessitam do apoio de novas soluções que diminuam ou controlem a hemorragia.
\end{abstract}

A utilização de hemostáticos tópicos é um conceito antigo, empregado em diferentes formas pelas civilizações antigas. O povo egípcio fazia uso de uma mistura de cera, graxa, e cevada, em um esforço para parar sangramentos. Na Grécia antiga, ervas ditas hemostáticas pelos padres e curandeiros da época eram aplicadas em ferimentos de guerra ${ }^{1}$. Recentemente, os avanços na biotecnologia resultaram no desenvolvimento de agentes hemostáticos tópicos que atualmente estão disponíveis para o cirurgião. Esses agentes vão desde hemostáticos tópicos absorvíveis, como gelatinas, colágeno microfibrilar e celulose oxidada regenerada, a hemostáticos tópicos biologicamente ativos, tais como trombina, colas biológicas e outros agentes combinados.

A grande variedade de hemostáticos tópicos somada à baixa frequência de artigos de revisão na literatura nacional sobre este tópico nos estimulou a elaborar este manuscrito. Uma revisão em bases eletrônicas PUBMED, EMBASE, MEDLINE e LILACS foi realizada buscando manuscritos nos idiomas inglês, português, francês e espanhol. Serão apresentados os agentes hemostáticos tópicos mais comumente utilizados em cirurgia, dividindo-os em absorvíveis, biológicos e sintéticos, conforme listados na tabela 1.

\section{AGENTES ABSORVÍVEIS}

\section{Celulose oxidada regenerada (Surgicel Original ${ }^{\circledR}$, Surgicel Nu-Knit ${ }^{\circledR}$, Surgicel Fibrillar ${ }^{\circledR}$, Interceed ${ }^{\circledR}$, Gelitacel $^{\circledR}$ )}

A celulose oxidada simples foi inicialmente proposta em 1942, nos Estados Unidos. Em 1960, um novo hemostático tópico - celulose oxidada

1 - Universidade de Campinas, Departamento de Cirurgia, Disciplina de Cirurgia do Trauma, Campinas, SP, Brasil. 
Tabela 1. Tipos, nome comercial e mecanismo de ação dos agentes hemostáticos tópicos.

\begin{tabular}{|c|c|c|}
\hline Hemostático tópico & Nome comercial & Mecanismo de ação \\
\hline Celulose oxidada regenerada & $\begin{array}{l}\text { Surgicel Original } ₫ \text {, Surgicel } \\
\text { Nu-Knit } ₫ \text {, Surgicel Fibrillar } ₫ \text {, } \\
\text { Interceed } ₫ \text {, Gelitacel } \circledast\end{array}$ & $\begin{array}{l}\text { Matriz física para iniciação do } \\
\text { coágulo. O baixo pH promove efeito } \\
\text { antimicrobiano }\end{array}$ \\
\hline Gelatinas & 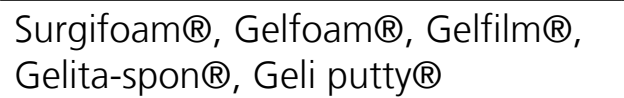 & $\begin{array}{l}\text { Matriz física para iniciação do } \\
\text { coágulo }\end{array}$ \\
\hline Gelatina + Trombina & FloSeal $®$, Surgiflo® & $\begin{array}{l}\text { Combinação de efeitos das } \\
\text { gelatinas e trombinas }\end{array}$ \\
\hline Colágeno microfibrilar & 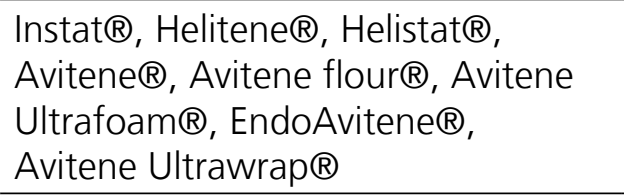 & Aderência e ativação plaquetária \\
\hline Selantes de fibrina & 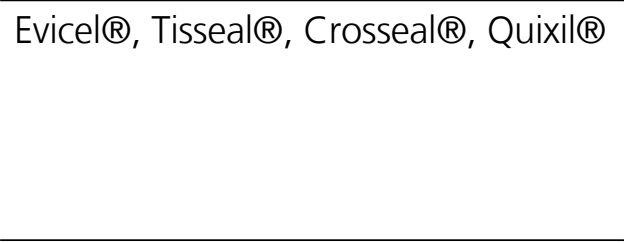 & $\begin{array}{l}\text { Trombina e fibrinogênio } \\
\text { combinados no momento da } \\
\text { aplicação. Trombina degrada } \\
\text { fibrinogênio em fibrina formando } \\
\text { coágulo }\end{array}$ \\
\hline Trombina tópica & $\begin{array}{l}\text { Evithrom®, Recothrom®, Thrombin- } \\
\text { JMI® }\end{array}$ & $\begin{array}{l}\text { Converte fibrinogênio em fibrina } \\
\text { para formar coágulo. Promove } \\
\text { ativação de fatores de coagulação }\end{array}$ \\
\hline $\begin{array}{l}\text { Adesivo de glutaraldeído e } \\
\text { albumina bovina }\end{array}$ & BioGlue® & $\begin{array}{l}\text { Gluteraldeído interage com } \\
\text { albumina bovina e proteína celular } \\
\text { no local da ferida para formar uma } \\
\text { resistente matriz }\end{array}$ \\
\hline Adesivos de cianoacrilato & Dermabond $®$, Omnex $®$ & $\begin{array}{l}\text { Monômeros líquidos formam } \\
\text { polímeros na presença de água e } \\
\text { colam duas superfícies }\end{array}$ \\
\hline Polietilenoglicol & CoSeal@ & $\begin{array}{l}\text { Dois polímeros de polietilenoglicol se } \\
\text { misturam e reagem no local da ferida }\end{array}$ \\
\hline
\end{tabular}

regenerada (COR) - surgiu no mercado com o lançamento do Surgice ${ }^{\circledR}$, o único até então não derivado de elementos humanos ou animais². A COR é, portanto, um hemostático tópico de origem vegetal manufaturado pela regeneração de celulose pura em tecido (algodão) entrelaçado que posteriormente é oxidado. Sua unidade funcional é o ácido anidroglucurônico. A celulose oxidada regenerada é de fácil aplicação e manipulação em cirurgia pela característica de malha entrelaçada frouxa que possui, lembrando um chumaço de algodão (Surgicel
Fibrillar ${ }^{\circledR}$, Gelitace ${ }^{\circledR}$ ) ou um pequeno pedaço de malha (Surgicel Nu-Knit ${ }^{\circledR}$ ), agindo assim como matriz de suporte para a iniciação e formação do coágulo. A celulose oxidada regenerada não é pegajosa, não adere ao material cirúrgico, pode ser moldado em diferentes formas e tamanhos, não desmancha na presença de líquidos e suporta compressão sem perder sua propriedade. Este material não deve ser infundido em meio aquoso previamente à utilização, uma vez que possui melhor atividade hemostática quando aplicado seco. 
Ambas as formas comerciais de COR diminuem o $\mathrm{pH}$ local uma vez aplicados. $\mathrm{O} \mathrm{pH}$ ácido provoca hemólise, o que explica a coloração amarronzada do material quando em contato com sangue. A hemoglobina resultante da hemólise reage com o pH local (ácido) formando hematina ácida. Uma das vantagens teóricas do baixo $\mathrm{pH}$ é a potencial ação antimicrobiana e ação cáustica, potencializando a hemostasia e a formação de um coágulo artificial1. O baixo pH local, entretanto, possui desvantagens, já que inativa a ação de coagulantes biologicamente ativos, como a trombina, impossibilitando, portanto, a utilização da COR em conjunto com outros agentes hemostáticos biológicos. Além disso, a natureza ácida da COR pode aumentar o processo inflamatório local e retardar o processo normal de cicatrização ${ }^{3}$. Outro ponto importante, que pode induzir a equipe cirúrgica a submeter seu paciente à intervenção desnecessária, e que também pode ser atribuído aos hemostáticos de base gelatinosa, é a aparência pós-operatória de uma imagem à tomografia com halo hiperdenso e centro hipodenso, que pode ser confundida com um abscesso. Imagens não comuns que geraram dúvida diagnóstica foram recentemente reportadas na literatura tanto em adultos quanto em pacientes pediátricos. Mais do que isso, complicações em cirurgia foram reportadas com o uso da COR em situações de hemorragia grave ${ }^{4,5}$. A dissolução da COR pode variar de duas semanas a alguns anos, dependendo da quantidade do produto utilizada ${ }^{3}$.

\section{Gelatinas (Surgifoam ${ }^{\circledR}$, Gelfoam ${ }^{\circledR}$, Gelfilm $^{\circledR}$, Gelita-spon ${ }^{\circledR}$, Geli putty ${ }^{\circledR}$, FloSeal $^{\circledR}$ )}

A gelatina é um agente hemostático manufaturado a partir do colágeno animal (derme ou tendão de porcino, ovelhas ou equinos) hidrolizado e purificado. Foi introduzida no mercado em 1945 e poucos avanços em sua forma e composição foram realizados desde então ${ }^{6}$. Apesar de seu mecanismo de ação não ser inteiramente conhecido, acredita-se que atue mais de forma física do que quimicamente na cascata de coagulação ${ }^{7}$. Assim, como no caso da $\mathrm{COR}$, as gelatinas são úteis como uma matriz física para iniciação do coágulo.

A matriz de gelatina pode ser utilizada em diferentes formas: esponjas, pó e solução fisiológica estéril, formando um aspecto espumoso quando misturados ou saturados em trombina purificada. A solução espumosa está associada à diminuição da infecção do sítio de aplicação e demonstrou ser útil na diminuição do sangramento de origem óssea, como, por exemplo, nas esternotomias e neurocirurgias.

Uma importante propriedade das diferentes formas de gelatina é sua habilidade de absorver mais de 40 vezes seu peso em sangue e fluidos e sua habilidade em expandir seu volume em $200 \%$ in vivo. Isto significa que as formas de gelatina aumentam mais seu volume do que os hemostáticos tópicos à base de colágeno e celulose oxidada regenerada e que, apesar desta propriedade fornecer boa ação mecânica hemostática, pode também, de certa forma, ser vista, em algumas especialidades, como um ponto negativo, especialmente quando usados em espaços confinados ou próximos de estruturas nervosas, gerando complicações de origem compressiva. O cirurgião geral e do trauma pode beneficiar-se dessa propriedade nos traumas penetrantes. Feridas transfixantes de órgãos sólidos, em particular, podem ser manejadas com este material isoladamente ou em conjunto com COR.

Raros relatos de complicação relacionada ao uso de gelatinas, como formação de abscessos ou granulomas, foram publicados na literatura. Alguns destes atribuídos à imagens radiológicas pós-operatórias duvidosas, mais tarde identificados como hemostáticos tópicos à base de gelatina ${ }^{8,9}$.

A matriz de gelatina é absorvida dentro de quatro a seis semanas e referida como não antigênica, embora seja derivada de produtos de origem animal. Diferentemente da celulose oxidada regenerada, o pH da matriz de gelatina é neutro e, portanto, pode ser 
usado em conjunto com a trombina ou outros agentes hemostáticos objetivando reforçar a ação hemostática. Acessibilidade, facilidade de utilizar, baixo preço e boa atividade hemostática fazem dos hemostáticos tópicos com matriz de gelatina uma ferramenta popular para redução da morbidade causada pela hemorragia.

Um grande avanço nos hemostáticos com matriz de gelatina veio com o desenvolvimento de um produto denominado FloSea ${ }^{\circledR}$, aprovado para uso comercial em 1999, nos EUA, e que merece ser colocado à parte nessa seção. Diferentemente dos outros hemostáticos tópicos com matriz de gelatina, o FloSeal ${ }^{\circledR}$ é uma combinação de matriz de gelatina à base de colágeno bovino contendo microgrânulos, reticulada com glutaraldeído (cola biológica) e solução de trombina humana, que são misturados no momento do uso ${ }^{10,11}$. Em contato com o sangue, as partículas da gelatina incham e induzem a um efeito de tamponamento. Esta característica permite que ele seja mais eficaz no controle do sangramento arterial moderado em comparação com os outros agentes. FloSeal ${ }^{\circledR}$ foi comprovadamente superior ao Gelfoamtrombina em cirurgias cardíacas e foi demonstrado conseguir reduzir o sangramento quando utilizado em nefrectomias abertas e por laparoscopia ${ }^{12}$. Apesar de não ser importante em determinadas intervenções cirúrgicas, a propriedade líquida do FloSeal ${ }^{\circledR}$ facilita a aplicação em determinados procedimentos e técnicas, especialmente na cirurgia minimamente invasiva. Foi demonstrado que o uso do FloSeal ${ }^{\circledR}$ em cirurgia é seguro e eficaz ${ }^{10,12}$.

\section{Colágeno Microfibrilar (Instat ${ }^{\circledast}$, Helitene ${ }^{\circledR}{ }$, Helistat $^{\circledR}$, Avitene $^{\circledR}$, Avitene flour $^{\circledR}$, Avitene Ultrafoam ${ }^{\circledR}$, EndoAvitene ${ }^{\circledR}$, Avitene Ultrawrap ${ }^{\circledR}$ )}

Desenvolvido e introduzido no mercado nos anos 1970, os hemostáticos tópicos à base de colágeno microfibrilar (CMF) são produzidos através da purificação das fibras de colágeno (proteína ressecada) de bovino, equino ou caprino e posteriormente processados em microcristais, na qual são manipulados em variadas formas comerciais. A marca Avitene ${ }^{\circledR}$ foi primeiramente lançada em forma de pó, e ainda é comumente utilizada. Todos os tipos de hemostáticos à base de colágeno ganharam uso precoce generalizado quando se percebeu que pareciam ser mais efetivos do que os hemostáticos tópicos à base de gelatina. Os produtos à base de colágeno ativam a via intrínseca da cascata de coagulação, enquanto os hemostáticos à base de gelatina induzem a hemostasia por meio de propriedades físicas. Em alguns ensaios clínicos randomizados, os hemostáticos de colágeno microfibrilar mostraram-se superiores à celulose oxidada regenerada e demonstraram ter redução estatisticamente significativa da perda sanguínea quando comparados entre $\mathrm{si}^{13}$. Os avanços e melhorias feitas no campo dos hemostáticos à base de colágeno têm sido no lançamento de novos formatos comerciais. O primeiro hemostático tópico à base de colágeno (Avitene ${ }^{\circledR}$ ) estava inicialmente disponível em forma de pó, e hoje a linha de produtos tem se expandido em materiais em forma de tecido, bem como, em formato de esponja e pequenos "pad's" ou placas (Instat $\left.{ }^{\circledR}\right)$.

O CMF fornece uma generosa área de superfície que, quando em contato com sangue, permite aderência e ativação plaquetária promovendo a formação do trombo dentro de dois a cinco minutos ${ }^{14}$. Considerando então, que o seu mecanismo de ação depende da ativação plaquetária, o CMF é menos eficaz em pacientes com trombocitopenia grave ou coagulopatias, no entanto, atinge com sucesso hemostasia mesmo em pacientes heparinizados. O CMF não aumenta seu volume, como é o caso das gelatinas, e é absorvido em menos de oito semanas. Deve ser aplicado em superfícies sangrantes com instrumento seco e não com as mãos do cirurgião, pois tende a aderir às luvas. Os hemostáticos à base de colágeno microfibrilar têm sido usados com sucesso no controle de áreas de sangramento extenso. Em procedimentos laparoscópicos, opções disponíveis no mercado (Endo-Avitene $\left.{ }^{\circledR}\right)$, que se caracterizam pelo formato de 
folhas laminadas, estão disponíveis com um aplicador que facilitam a introdução através de trocarteres. Tal como acontece com a celulose oxidada regenerada, recomenda-se a remoção do excesso de CMF do sítio cirúrgico após hemostasia adequada, uma vez que podem se ligar à estruturas neurais e causar dor ou dormência. Infecções do sítio da aplicação do hemostático tópico à base de CMF foram descritas como mais frequentes quando comparadas aos hemostáticos à base de COR. Complicações e dúvidas em exames de imagem relacionadas a procedimentos em Neurocirurgia foram amplamente reportados, levando pesquisadores a realizarem estudos de segurança e eficácia em animais, especificamente nesta área cirúrgica ${ }^{15}$.

\section{AGENTES BIOLÓGICOS}

\section{Selantes de fibrina $\left(\right.$ Evicel $^{\oplus}$, Tisseal $^{\oplus}$, Crosseal $^{\oplus}$, Quixil ${ }^{\oplus}$ )}

O selante de fibrina foi citado pela primeira vez na literatura em 1909, na Europa. Estudos foram retomados nos anos 1940, após a disponibilização de trombina purificada e sua utilização se tornou comum em diferentes especialidades cirúrgicas, inclusive durante a Primeira Grande Guerra. Nos EUA, foi aprovado para uso em humanos somente nos anos 1980 (Tisseal ${ }^{\circledR}$ ), após comprovação de ser seguro e eficaz ${ }^{16}$.

O clássico selante de fibrina consiste em fibrinogênio humano agrupado, liofilizado, e trombina bovina ou humana, algumas vezes também contendo fator de coagulação XIII concentrado e aprotinina ${ }^{17}$. O fator XIII é uma pró-enzima que é ativada pela trombina na presença de íons cálcio (fator estabilizador de fibrina). Uma vez ativado, o fator XIII forma ligações cruzadas entre cadeias de fibrina, estabilizando a formação de coágulos. Sendo o mais antigo tipo de cola no mercado, variações de múltiplos produtos estão disponíveis, com concentrações variáveis dos seus ingredientes. Isso modifica as propriedades de coagulação. A resistência à tração de coágulos de sangue é uma função de vários fatores e a concentração de fibrinogênio é de grande importância. A trombina tem um impacto principalmente na velocidade e na integridade de coagulação e, indiretamente, sobre a estabilidade do coágulo ${ }^{18}$. A aprotinina é um inibidor de protease (tecido de pulmão bovino) que inibe a tripsinina, plasmina e calicreínas, retardando a lise de coágulos mediada por plasmina. As colas de fibrina são aplicadas através de uma técnica de seringa dupla. A aplicação ideal requer um campo operatório seco. As colas de fibrina são particularmente eficazes quando aplicados antes do sangramento. Nesta situação, o fibrinogênio pode polimerizar antes que a pressão arterial aumente o fluxo da microcirculação local. Quando usado após o início do sangramento, pressão local deve ser aplicada sobre a ferida para permitir a polimerizaçãa ${ }^{19}$.

A combinação com celulose, gelatina e/ ou colágeno (lã, mantas ou outras matrizes) com substâncias pró-coagulantes, seja fibrina ou trombina, é uma abordagem criada recentemente (Surgiflo ${ }^{\oplus}$ : gelatina + trombina) para aumentar a eficácia dos hemostáticos tópicos, sendo classificado como selante de fibrina com matriz sólida. Estes produtos combinam os efeitos mecânicos de um tamponamento temporal com os efeitos hemostáticos do selante de fibrina. A matriz de colágeno induz a agregação de plaquetas e estimula a coagulação do fator XII. Em contato com líquido, os componentes sólidos se dissolvem e formam um coágulo de fibrina viscoso entre a matriz e a superfície cruenta da ferida. Um período de compressão é necessário para que ocorra polimerização dos componentes do selante.

Hemostáticos tópicos de fibrina que não necessitam de compressão podem ser obtidos com a ajuda de partículas que absorvem líquidos e aumentam de tamanho, dissolvidas em matriz líquida, e aderem às feridas cirúrgicas (FloSeal ${ }^{\circledR}$ ). São classificados como selante de fibrina com matriz líquida. Este material não necessita de campo cirúrgico seco para aplicação. Outros produtos $\left(\right.$ CoStasis $^{\circledast}$, Vitage $\left.^{\circledR}\right)$ aprovados para uso em 
humanos em 2000, nos EUA, são constituídos por um composto de colágeno microfibrilar e trombina bovina, misturadas em uma seringa com plasma (autólogo que contém fibrina e plaquetas) do paciente retirado e centrifugado durante a cirurgia ${ }^{1}$. Os componentes do plasma fornecem o fibrinogênio, que é clivado pela trombina para formar uma matriz gelatinosa de colágeno-fibrina. Como os outros hemostáticos locais combinados, é um produto caro e o sucesso do seu uso depende da experiência do cirurgião $0^{1,20}$.

Em 2003, o Crosseal $^{\circledR}$ foi introduzido pela Cruz Vermelha norte americana. É composto apenas por produtos de origem humana (fibrinogênio e trombina agrupada do plasma, bem como, ácido tranexâmico) ${ }^{21}$. Em 2004, Schwartz et al. ${ }^{22}$ reportaram, em estudo multicêntrico prospectivo randomizado, o Crosseal $^{\circledR} / \mathrm{Quixi}{ }^{\circledR}$ como o mais efetivo hemostático, com menor tempo para hemostasia efetiva, menor sangramento intraoperatório e menor indução de complicações quando comparado ao grupo controle (Avitene ${ }^{\circledR}$, Surgice ${ }^{\circledR}$, Surgicel NuKnit ${ }^{\circledR}$, Gelfoam, Gelfoam + trombina)

Outro agente que entrou no mercado mais recentemente foi o Evicel ${ }^{\circledR}$, que não contém inibidores fibrinolíticos e requer um tempo mínimo de preparação antes da aplicação, tornando-o de mais fácil utilização. Pode ser usado na forma de spray, apresentando hemostasia satisfatória em superfícies cruentas, como aquelas resultantes de trauma hepático. Em nossa experiência, as colas de fibrina Tissee ${ }^{\circledR}$, Quixil ${ }^{\circledR}$ e Evice ${ }^{\circledR}$ são especialmente adequadas para controlar sangramentos de baixa pressão (venoso) e de superfícies cruentas, como dos rins, fígado e baço.

Inicialmente, as aplicações clínicas para os selantes de fibrina foram em grande parte limitadas à Cirurgia Cardíaca, Cirurgia Vascular, Cirurgia Oral e Maxilofacial e Cirurgia Plástica Reconstrutiva, mas, recentemente várias aplicações estão sendo discutidas na literatura para outras Especialidades, como na Cirurgia do Trauma, Urologia, Obstetrícia e Neurocirurgia. Na área da Cirurgia do Trauma, especificamente, alguns autores demonstraram que o uso de selantes de fibrina em traumas hepáticos graves excluíram a necessidade de empacotamento do órgão23,24.

Apesar do selante de fibrina ter sido um dos primeiros agentes hemostáticos a ser utilizado na prática clínica, o lento desenvolvimento da tecnologia, os obstáculos iniciais para a aprovação pelo FDA (Food and Drug Administration), nos EUA, e sua limitada disponibilidade subsequente, levaram a uma relativa escassez de investigação clínica prospectiva com selantes de fibrina. Sendo material com grande potencial de utilização e relativamente pouco estudado, os selantes de fibrina tiveram um espaço interessante para novas pesquisas em cirurgia. Uma nova forma comercial de selante de fibrina que foi recentemente aprovado para uso em humanos pelo FDA, após diversos estudos em modelos experimentais, foi o TachoSil ${ }^{\circledR(25-27}$, que lembra uma placa esponjosa e demonstrou sua utilidade não apenas como hemostático, mas também em anastomoses intestinais e em fístulas traqueobrônquicas, devido a sua alta capacidade adesiva ${ }^{25,28}$. Até o momento, nenhum efeito adverso foi relacionado ao uso do TachoSil ${ }^{\circledR}$, apesar de haver relatos na literatura sobre a presença de anticorpos após a aplicação do produto, porém sem expressão clínica ${ }^{27}$.

\section{Trombina tópica (Evithrom ${ }^{\circledR}$, Recothrom ${ }^{\circledR}$ Thrombin-JMI®)}

A trombina é uma enzima natural com papel na hemostasia, inflamação e sinalização celular. É formada a partir da pró-trombina, como resultado da ativação das vias de coagulação intrínsecas e extrínsecas, e forma a base do coágulo de fibrina, promovendo a conversão do fibrinogênio em fibrina. A trombina já foi purificada a partir de várias fontes e usada como auxiliar na hemostasia tópica por mais de 60 anos. Até recentemente, a única trombina disponível comercialmente era derivada do plasma bovino (trombina-JMI). Apesar de a 
trombina ter sido utilizada como uma ferramenta eficaz para parar o sangramento, a trombina de origem bovina, mostrou induzir uma resposta imune importante ${ }^{29}$. Diversos estudos têm documentado uma série de eventos clínicos que acompanham a exposição humana à trombina bovina, e incluem o desenvolvimento de anticorpos contra a trombina, protrombina, fator $\mathrm{V}$ e cardiolipina ${ }^{29}$. Pacientes em hemodiálise, com elevados níveis de anticorpos contra a trombina bovina tópica, também foram observados por terem aumento da incidência de trombose do acesso vascular, coagulopatia grave e sangramento após a exposição à trombina bovina ${ }^{30}$.

Devido a essas preocupações, pesquisadores desenvolveram a trombina derivada de plasma humano e a trombina humana recombinante. Em 2007, Bowman et al. ${ }^{31}$ demonstraram, em estudo comparativo entre trombina recombinante humana tópica e trombina bovina, que a trombina recombinante humana demonstrou a mesma eficácia na hemostasia cirúrgica, perfil de segurança semelhante e resposta imunológica significativamente menor do que a trombina de bovinos.

\section{AGENTES SINTÉTICOS}

\section{Adesivo de glutaraldeído e albumina bovina (BioGlue ${ }^{\circledR}$ )}

O adesivo de glutaraldeído e albumina bovina ( $A G A B$ ) consiste em uma solução de glutaraldeído a $10 \%$ e $45 \%$ de solução de albumina bovina, purificada por precipitação, cromatografia por calor e radiação ${ }^{32}$. Foi aprovada para uso, nos EUA, em 1999, sendo apresentada comercialmente em uma seringa com dois compartimentos separados e um mesmo bico dispensador, quando, então, seus componentes são misturados no momento da aplicação. Em um prazo de 20 segundos o adesivo tem $65 \%$ de potência de ligação, e obtém sua plena força de ligação em dois minutos, independentemente da temperatura ou do meio de aplicação (ar ou água) ${ }^{33}$. Num estudo em que o BioGlue ${ }^{\circledast}$ foi utilizado como agente hemostático em 79 cirurgias cardíacas, houve sucesso em 78 casos.

Apesar de algumas pesquisas na área da cirurgia cardíaca sugerirem o uso de BioGlue ${ }^{\circledR}$ em suturas do pericárdio e próteses vasculares, sua indicação ainda parece controversa neste e em outros segmentos da cirurgia ${ }^{33,34}$. Recentemente, um grupo da Áustria realizou um interessante estudo experimental comparando o uso de $A G A B$ e o selante de fibrina nas cirurgias de hérnia inguinal. Segundo os autores, o AGAB demonstrou boa potência de ligação, porém baixa biocompatibilidade (citotoxidade). Persistente atividade inflamatória foi evidenciada, não favorecendo, portanto, o uso deste material nesta indicação específica ${ }^{35}$. Persistente reação inflamatória resultando em estenose também foi relatada em estudo experimental com porcos jovens em reforços anastomóticos aorto-aórticos, comprometendo sua indicação em crianças e jovens ${ }^{36}$. Por outro lado, outra análise experimental sugere a utilização do AGAB como reforço das anastomoses gastrointestinais realizadas por grampeadores, no entanto, afirmam que mais estudos são necessários para determinar se o AGAB é ou não capaz de prevenir ou ajudar nos casos de fístulas anastomóticas ${ }^{37}$. Complicações e interpretações duvidosas em métodos de imagem também são reportadas na literatura em diversas áreas cirúrgicas, incluindo a Cirurgia Cardiotorácica e a Neurocirurgia ${ }^{38}$.

\section{Adesivos de cianoacrilato (Dermabond ${ }^{\circledR}$, Omnex $^{\circledR}$ )}

Desenvolvidos em 1942 pelo químico Harry Coover $^{39}$ e, posteriormente, comercializado como Super Bonder, os cianoacrilatos são monômeros líquidos que rapidamente formam polímeros na presença de água (íons hidroxil) e, assim, rapidamente colam superfícies adjacentes. A 
propriedade de cola instantânea tornou promissor o uso dos cianoacrilatos como adesivos e agentes hemostáticos, existindo relatos de que, durante a Guerra do Vietnã, cianoacrilatos foram utilizados sobre feridas abertas sangrantes em um esforço para retardar a hemorragia enquanto o soldado ferido era transportado para um centro médico próximo.

O cianocrilato de etila, como Super Bonder, demonstrou-se citotóxico durante sua degradação (cianoacetato e formaldeído) resultando em uma resposta inflamatória acentuada e persistente, retardando a síntese e a cura da ferida. Após uma reestruturação da molécula em 2-octil-cianoacrilato, o produto recebeu a aprovação do FDA com indicação de uso no reparo de feridas, comercializado como Dermabond $^{\circledR}$. O 2-octil-cianoacrilato atinge sua força máxima de ligação em 2,5 minutos de aplicação e forma uma ligação mais forte e mais flexível do que o 2-butil-cianoacrilato'. Na Ásia, o 2-butil-cianoacrilato também encontrou aplicação para embolização de hemorragias gástricas pela injeção direta do adesivo no vaso sangrante ${ }^{1,40}$. Apesar do uso do produto em varizes gastresofágicas ter demonstrado resultado satisfatório na hemostasia rápida na maioria dos casos, sua utilização incorre em risco de trombose da veia mesentérica ${ }^{40}$. Relatos de casos descrevem o uso do 2-octil-cianoacrilato na radiologia intervencionista para embolização de shunts de veias porto-sistêmicas e pseudoaneurismas anastomóticos ${ }^{41}$. Complicações relacionadas à embolização também foram descritas e sua indicação parece ser controversa ${ }^{42}$. As vantagens de cianoacrilatos para reparação de tecidos incluem, apesar do custo, uma aplicação e tempo de reparo mais rápidos com resultado cosmético equivalente aos resultados da sutura para as incisões e feridas pequenas, sem a eventual necessidade de uma consulta de acompanhamento para a remoção da sutura.

Outro produto à base de cianoacrilato e indicado para uso em reconstruções e anastomoses vasculares é o Omnex ${ }^{\circledR}$. Embora existam ainda poucos estudos sobre o uso do produto, o Omnex ${ }^{\circledR}$ possui segurança e eficácia comprovadas e tem se mostrado interessante para os cirurgiões vasculares e cardiotorácicos, por ser fácil de utilizar, atingir hemostasia imediata quando aplicado e agir independentemente do sistema de coagulação ${ }^{43}$. Adesivos à base de cianoacrilato não podem ser utilizados para aproximar feridas com perda tecidual, lesões das articulações, mãos ou pés, ou para reparar superfícies mucosas dilaceradas ${ }^{44}$. Os cianoacrilatos estão se tornando cada vez mais populares para a sutura de pequenas incisões na pele, desde que esta seja uma aproximação livre de tensão. E, como cirurgiões de diferentes áreas vêm se tornando cada vez mais confortáveis com as propriedades desses adesivos, os cianoacrilatos estão sujeitos a usos mais inovadores e a motivo de pesquisa.

\section{Polietilenoglicol (CoSeal $\left.{ }^{\circledR}\right)$}

CoSeal $^{\circledR}$ é um polímero totalmente sintético utilizado por cirurgiões para reparar áreas de potenciais sangramentos em anastomoses vasculares. Utiliza dois polietilenoglicois sintéticos que, uma vez misturados, podem ser aplicados diretamente às superfícies dos tecidos ou usado para selar as linhas de sutura ou enxertos sintéticos. O selante funciona interagindo os produtos sintéticos com o tecido humano. O polímero permanece flexível e é reabsorvido pelo organismo em cerca de quatro semanas, segundo o fabricante. O CoSeal ${ }^{\circledR}$ pode aumentar em até quatro vezes o seu tamanho original. Um estudo conduzido pela Associação Europeia de Cirurgia Cardiotorácica evidenciou que pacientes que receberam uma grande aplicação de CoSeal $^{\circledR}$ evoluíram com tamponamento cardíaco. Oclusão da veia cava superior ocorreu como resultado direto do uso CoSeal ${ }^{\circledR}$ em um dos 76 pacientes estudados ${ }^{45}$. Neste mesmo estudo, - CoSeal ${ }^{\circledR}$ se mostrou útil para a prevenção de aderências do pericárdio em pacientes que possam necessitar de reintervenção cirúrgica, especialmente em crianças que sofrem de cardiopatias congênitas. 
O polietilenoglicol é um agente eficaz para a hemostasia vascular e cardíaca ou em aplicações cirúrgicas em que a expansão do volume do produto não é uma preocupação. Seu desempenho na vedação anastomótica é equivalente ao Gelfoam com trombina, mas a principal vantagem do CoSeal ${ }^{\circledR}$ reside na rapidez com que atinge a hemostasia.

\section{CONSIDERAÇÕES FINAIS}

Diante da heterogênea família de hemostáticos tópicos, a decisão sobre que hemostático tópico utilizar e quando, se torna por muitas vezes confusa. Um melhor entendimento dos diversos tipos de hemostáticos tópicos e a experiência prática adquirida permitirão ao cirurgião formar um adequado senso crítico para indicar o agente correto, no momento adequado. O agente ideal é aquele fácil de usar, eficaz, utilizável em qualquer ou na maioria das condições cirúrgicas, não antigênico, totalmente absorvível e com custo acessível. Acreditamos que o avanço da biociência e a realização de novas pesquisas experimentais e clínicas definirão o agente hemostático de melhor custo benefício.

\section{A B S T R A C T}

Hemostasis plays a critical and fundamental role in all surgical procedures. Its management has several key points that start with good operative technique and adequate anesthetic support. Certain situations, such as severe bleeding resulting from penetrating trauma, do not depend exclusively on the control of the surgical team and require the support of new solutions that decrease or control bleeding. Since ancient times, a hallmark of medicine has been to act in the control of hemorrhage, and more recently, in the facilitation of hemostasis by the application of topical agents by either manual compression or modern agents. In the last decade, the number of different topical hemostatic agents has grown dramatically. For the modern surgeon to choose the right agent at the right time, it is essential that he/she understands the mechanisms of action, the effectiveness and the possible adverse effects related to each agent. Thus, the great variety of topical hemostatics, coupled with the absence of a review article in the national literature on this topic, stimulated us to elaborate this manuscript. Here we report a detailed review of the topical hemostatic agents most commonly used in surgical specialties.

Keywords: Hemostatic. General Surgery. Hemostasis. Surgical. Hemostatic Techniques. Surgical Procedures. Operative.

\section{REFERÊNCIAS}

1. Achneck HE, Sileshi B, Jamiolkowski RM, Albala DM, Shapiro ML, Lawson JH. A comprehensive review of topical hemostatic agents: efficacy and recommendations for use. Ann Surg. 2010;251(2):217-28.

2. Sundaram CP, Keenan AC. Evolution of hemostatic agents in surgical practice. Indian J Urol. 2010;26(3):374-8.

3. Tomizawa Y. Clinical benefits and risk analysis of topical hemostats: a review. J Artif Organs. 2005;8(3):137-42.

4. Alves Júnior L, Vicente $W V$, Ferreira CA, Manso $\mathrm{PH}$, Arantes LR, Pinheiro KS, et al. Surgicel packing and an erroneous diagnosis of mediastinitis in a neonate. Tex Heart Inst J. 2010;37(1):116-8.

5. Brodbelt AR, Miles JB, Foy PM, Broome JC. Intraspinal oxidised cellulose (Surgicel) causing delayed paraplegia after thoracotomy--a report of three cases. Ann R Coll Surg Engl. 2002;84(2):97-9.
6. Schonauer C, Tessitore E, Barbagallo G, Albanese $\mathrm{V}$, Moraci $\mathrm{A}$. The use of local agents: bone wax, gelatin, collagen, oxidized cellulose. Eur Spine J. 2004;13 Suppl 1:S89-96.

7. Sabel $M$, Stummer $W$. The use of local agents: Surgicel and Surgifoam. Eur Spine J. 2004;13 Suppl 1:S97-101.

8. Kawano H, Arakawa S, Satoh O, Matsumoto Y, Hayano M, Miyabara S. Foreign body granulomatous change from absorbable gelatin sponge and microcoil embolization after a guidewire-induced perforation in the distal coronary artery. Intern Med. 2010;49(17):1871-4.

9. Sandrasegaran K, Lall C, Rajesh A, Maglinte DT. Distinguishing gelatin bioabsorbable sponge and postoperative abdominal abscess on CT. AJR Am J Roentgenol. 2005;184(2):475-80.

10. Oz MC, Rondinone JF, Shargill NS. FloSeal Matrix: new generation topical hemostatic sealant. J Card Surg. 2003;18(6):486-93. 
11. Reuthebuch $O$, Lachat $M L$, Vogt $P$, Schurr $U$, Turina $M$. FloSeal: a new hemostyptic agent in peripheral vascular surgery. Vasa. 2000;29(3):204-6.

12. Guzzo TJ, Pollock RA, Forney A, Aggarwal P, Matlaga BR, Allaf ME. Safety and efficacy of a surgeonprepared gelatin hemostatic agent compared with FloSeal for hemostasis in laparoscopic partial nephrectomy. J Endourol. 2009;23(2):279-82.

13. De Oliveira FM, de Carvalho MVH, Marchi E, Pinto CAL. Collagen, fibrinogen and thrombin biological addesive is effective in treating experimental liver injuries. Rev Col Bras Cir. 2016;43(4):254-61.

14. Wagner WR, Pachence JM, Ristich J, Johnson PC. Comparative in vitro analysis of topical hemostatic agents. J Surg Res. 1996;66(2):100-8.

15. Apel-Sarid L, Cochrane DD, Steinbok P, Byrne AT, Dunham C. Microfibrillar collagen hemostatinduced necrotizing granulomatous inflammation developing after craniotomy: a pediatric case series. J Neurosurg Pediatr. 2010;6(4):385-92.

16. de Carvalho MV, Marchi E, Pantoroto M, Rossini M, da Silva DM, Teodoro LF, et al. [Topical haemostatic agents and tissue adhesives]. Rev Col Bras Cir. 2013;40(1):66-71. Portuguese.

17. Spotnitz WD. Fibrin sealant: past, present, and future: a brief review. World J Surg. 2010;34(4):632-4.

18. Li J, Li HB, Zhai XC, Qin-Lei, Jiang XQ, Zhang ZH. Topical use of topical fibrin sealant can reduce the need for transfusion, total blood loss and the volume of drainage in total knee and hip arthroplasty: a systematic review and meta-analysis of 1489 patients. Int J Surg. 2016;36(Pt A):127-37.

19. Kraus TW, Mehrabi A, Schemmer P, Kashfi A, Berberat $P$, Buchler MW. Scientific evidence for application of topical hemostats, tissue glues, and sealants in hepatobiliary surgery. J Am Coll Surg. 2005;200(3):418-27.

20. Palm MD, Altman JS. Topical hemostatic agents: a review. Dermatol Surg. 2008;34(4):431-45.

21. Schexneider KI. Fibrin sealants in surgical or traumatic hemorrhage. Curr Opin Hematol. 2004;11(5):323-6.

22. Schwartz M, Madariaga J, Hirose R, Shaver TR, Sher $L$, Chari $R$, et al. Comparison of a new fibrin sealant with standard topical hemostatic agents. Arch Surg. 2004;139(11):1148-54.
23. Cohn SM, Feinstein AJ, Nicholas JM, McKenney MA, Sleeman D, Ginzburg E, et al. Recipe for poor man's fibrin glue. J Trauma. 1998;44(5):907.

24. Feinstein AJ, Varela JE, Cohn SM, Compton RP, McKenney MG. Fibrin glue eliminates the need for packing after complex liver injuries. Yale J Biol Med. 2001;74(5):315-21.

25. Marta GM, Facciolo F, Ladegaard L, Dienemann $H$, Csekeo A, Rea F, et al. Efficacy and safety of TachoSil $\circledast$ versus standard treatment of air leakage after pulmonary lobectomy. Eur J Cardiothorac Surg. 2010;38(6):683-9.

26. Fischer $L$, Seiler $C M$, Broelsch $C E$, de Hemptinne $\mathrm{B}$, Klempnauer J, Mischinger HJ, et al. Hemostatic efficacy of Tachosil in liver resection compared with argon beam coagulator treatment: An open, randomized, prospective, multicenter, parallelgroup trial. Surgery. 2011;149(1):48-55.

27. Rickenbacher $A$, Breitenstein $S$, Lesurtel $M$, Frilling A. Efficacy of TachoSil a fibrin-based haemostat in different fields of surgery--a systematic review. Expert Opin Biol Ther. 2009;9(7):897-907.

28. Pantelis D, Beissel A, Kahl P, Wehner S, Vilz TO, Kalff $J C$. The effect of sealing with a fixed combination of collagen matrix-bound coagulation factors on the healing of colonic anastomoses in experimental high-risk mice models. Langenbecks Arch Surg. 2010;395(8):1039-48.

29. Lawson JH, Lynn KA, Vanmatre RM, Domzalski $T$, Klemp KF, Ortel TL, et al. Antihuman factor $V$ antibodies after use of relatively pure bovine thrombin. Ann Thorac Surg. 2005;79(3):1037-8.

30. Lo CY, Jones C, Glader B, Zehnder JL. Development of antibodies to human thrombin and factor $\mathrm{V}$ in a pediatric patient exposed to topical bovine thrombin. Pediatr Blood Cancer. 2010;55(6):1195-7.

31. Bowman LJ, Anderson CD, Chapman WC. Topical recombinant human thrombin in surgical hemostasis. Semin Thromb Hemost. 2010;36(5):477-84.

32. Biggs $G$, Hafron J, Feliciano J, Hoenig DM. Treatment of splenic injury during laparoscopic nephrectomy with BioGlue, a surgical adhesive. Urology. 2005;66(4):882.

33. Passage J, Jalali H, Tam RK, Harrocks S, O'Brien MF. BioGlue Surgical Adhesive--an appraisal of its indications in cardiac surgery. Ann Thorac Surg. 2002;74(2):432-7. 
34. Coselli JS, Bavaria JE, Fehrenbacher J, Stowe CL, Macheers SK, Gundry SR. Prospective randomized study of a protein-based tissue adhesive used as a hemostatic and structural adjunct in cardiac and vascular anastomotic repair procedures. J Am Coll Surg. 2003;197(2):243-52; discussion 252-3.

35. Gruber-Blum S, Petter-Puchner AH, Mika K, Brand $J$, Redl $\mathrm{H}$, Ohlinger $W$, et al. A comparison of a bovine albumin/glutaraldehyde glue versus fibrin sealant for hernia mesh fixation in experimental onlay and IPOM repair in rats. Surg Endosc. 2010;24(12):3086-94.

36. LeMaire SA, Schmittling ZC, Coselli JS, Undar A, Deady BA, Clubb FJ Jr, et al. BioGlue surgical adhesive impairs aortic growth and causes anastomotic strictures. Ann Thorac Surg. 2002;73(5):1500-5; discussion 1506.

37. Nandakumar G, Richards BG, Trencheva K, Dakin G. Surgical adhesive increases burst pressure and seals leaks in stapled gastrojejunostomy. Surg Obes Relat Dis. 2010;6(5):498-501.

38. Babin-Ebell J, Bougioukakis $P$, Urbanski P, Froehner $S$, Diegeler A. Foreign material reaction to BioGlue(R) as a possible cause of cardiac tamponade. Thorac Cardiovasc Surg. 2010 Dec;58(8):489-91.

39. Hayes SC. Discovery of Super Glue helped land Coover in National Inventors Hall of Fame. Kingsport Times-News. 2004.

40. Belletrutti PJ, Romagnuolo J, Hilsden RJ, Chen F, Kaplan B, Love J, et al. Endoscopic management of gastric varices: efficacy and outcomes of gluing with $\mathrm{N}$-butyl-2-cyanoacrylate in a North American patient population. Can J Gastroenterol. 2008;22(11):931-6.
41. Yoshimatsu R, Takeuchi Y, Morishita H, lida N, Okabe $H$, Yamagami $T$, et al. Successful embolisation of intrahepatic portosystemic venous shunt using coils and n-butyl cyanoacrylate through two approach routes. Br J Radiol. 2006;79(947):e162-5.

42. Marine L, Gupta R, Gornik HL, Kashyap VS. Glue embolus complicating the endovascular treatment of a patient with Loeys-Dietz syndrome. J Vasc Surg. 2010;52(5):1350-3.

43. Brunkwall J, Ruemenapf G, Florek HJ, Lang W, Schmitz-Rixen T. A single arm, prospective study of an absorbable cyanoacrylate surgical sealant for use in vascular reconstructions as an adjunct to conventional techniques to achieve haemostasis. J Cardiovasc Surg (Torino). 2007;48(4):471-6.

44. Reckers LJ, Fagundes DJ, Cohen M. The ineffectiveness of fibrin glue and cyanoacrylate on fixation of meniscus transplants in rabbits. Knee. 2009;16(4):290-4.

45. Pace Napoleone C, Valori A, Crupi G, Ocello S, Santoro $F$, Vouhé $P$, et al. An observational study of CoSeal for the prevention of adhesions in pediatric cardiac surgery. Interact Cardiovasc Thorac Surg. 2009;9(6):978-82.

Recebido em: 22/04/2018

Aceito para publicação em: 28/08/2018

Conflito de interesse: nenhum.

Fonte de financiamento: nenhuma.

\section{Endereço para correspondência:}

Bruno Monteiro Pereira

E-mail: dr.bruno@gruposurgical.com.br 